digestive function is more or less impaired. In health the result of the untiring efforts of Boas to gain posthere should be nothing but what appears after a itive diagnostic means, in cases where cancer must regular Ewald test meal. If casein and meat are be suspected, but no tumor can be palpated. undigested and the latter even apparently hardened, you know that there is sub- or anacidity and impaired albuminous digestion; if there are mostly pieces of toast, you have hyperacidity and impaired starchy digestion. The small quantity of stomach contents gained, shows before filtration every other abnormal addition, such as blood, surplus of mucus, bile, etc.

You can use red and blue litmus paper, Congo paper, and the Dimethylamidoazobenzol test. You can then gain enough filtrate to determine the degree of acidity, and to try the Uffelmann carbolic acid and chlorid of iron test for lactic acid, the phloroglucinvanillin test for even an approximative, quantitative muriatic acid, the biuret reaction for a peptone and propeptone test, the iodin test to prove the absence or presence of starch and erythrodextrin, and a few drops for a rennet, or rennet zymogen test, and even a few drops to pour on a small piece of the white of an egg (hard boiled), or fibrin, for the pepsin test. You can do all that is generally done after a regular test meal, and have a perfect picture of the previous, more complicated digestion as well, especially for the estimation of the muscular power.

I have repeatedly convinced myself with others, as well as with my own stomach, that the tube always brings up portions of the first meal if any are still present in the stomach.

In introducing the tube a second time, pressing out every trace of the stomach contents, and using lavage until clear water appeared, I could always convince myself that the small quantity contained in the tube was a true representative of the entire contents of the stomach at the time. Nothing else could be expected, for we know from Beaumont's experiments with his Canadian, that those food particles which are not in proper condition to pass through the elective pylorus, either in consequence of chemical or other abnormities, are thrown back into the stomach, even up to the cardia.

This fact is quite in accordance with the daily experience of eructations of food particles, which, indigestible pro-tempore, of ten show the taste of an article taken with a former and not the latest meal.

It is easily understood that the first meal will per. mit different modifications, and may, for instance, consist of rice, soft-boiled eggs, meat and milk or water; provided that it consists of articles of food which in health will disappear within a given time, and which will at the same time represent the albuminous, starchy and fatty types of food, in a way that the particles which remain undigested will show a conspicuous difference from the second meal, and not be large enough to obstruct the eyes of the tube.

It is clearly demonstrated that an amount of material fills the stomach tube when the latter reaches the fundus, which is sufficient for all clinical purposes, especially for the demonstration of the motor, as well as of the digestive faculties of the stomach, and that by this method these results are reached in a way the least troublesome and dangerous for the patient, and most convenient for the examiner, whether he makes his investigations from a physiologic or pathologic standpoint.

I can not close these remarks on test meals without :alluding to a very important modification, which is
He advises lavage in the morning, until the water becomes perfectly clear without any reaction whatever, then a test breakfast of a soup prepared of one tablespoonful of flour free from every trace of lactic acid and not apt to form it. He designates a special kind of oatmeal (Hafermehl) for this purpose. The amount of water to be used in preparing the soup he gives at 500 c.c.; after one-half hour (the average time in his experiments) the contents of the stomach are aspirated and examined. With these precautions, and only then, he found lactic acid present (with but very few exceptions) only in cases of malignant disease.

\section{SPASMODIC ASTHMA CAUSED BY AORTIC ANEURISM.}

BY GEORGE TULLY VAUGHAN, M.D. PASSED ASSISTANT U. S. MARINE HOSPITAJ SERVICE.

It seems to me that a form of dyspnea, which closely resembles spasmodic asthma and from which it seems at times impossible to differentiate it, as a symptom in aortic aneurism, is important enough to deserve especial notice. In the three cases I am about to report it was a prominent and distressing symptom while other symptoms of aneurism were rather obscure. Asthma from aneurism is no doubt caused by pressure on the pneumogastric nerve and is not always continuous as might be supposed, for in one of my cases the patient had only three attacks in all and these occurred at irregular intervals during the last two months of his life. In the other cases this symptom was continuous but with marked remissions.

In obstinate and persistent cases of asthma in elderly persons, aneurism should always be born in mind as a possible cause, for in the absence of bruit, im. pulse, swelling, or circulatory disturbances it is not always easy to make a correct diagnosis. The pressure symptoms which cause changes in the pupil, paralysis of a vocal cord or unilateral sweating are not sufficient, as any other mediastinal tumor may produce the same effects.

"Tracheal tugging," first described by SurgeonMajor Porter of the British Army is a symptom of great value. It is brought out by taking hold of the cricoid cartilage when the patient is sitting with the neck relaxed, and putting the trachea slightly on the stretch. If there is aneurism of the arch, a distinct tugging of the trachea is felt with each pulsation of the heart.

(yase 1.-J. F. C., aged 60 years, native of Maine, was ad mitted to the Marine Hospital at Boston, Mass., June 14, 1888, with this history: Was first taken a year before with pain in left side of the chest from breast to back and in the left shoulder. Slight cough and once expectoration of a little red blood. Felt well after this until winter, when, after "taking cold," the pain and cough returned, there was nausea, unpleasant symptoms after eating, and loss of flesh. In appearance the patient was thin, with unhealthy color of skin, suggesting malignant cachexia.

Respiration in the left lung was weaker than in the right, but the heart seemed normal and no bruit nor tumor could be de tected. There was little change, patient expectorated blood two or three times, until about two months before death when he was suddenly taken with extreme dyspnea with all the signs of constriction of the bronchial tubes on both sides, râles, wheezing and orthopnen, lasting an hour or two when it was in a great measure relieved by morphia. 
In a day or two he was about as usual until the next attack, having in all three severe attacks and dying in the third.

Necropsy. ... Left lung weighed 170 grams, was small, atrophied and the seat of a low grade of inflammation; its pleural cavity was obliterated by adhesion. Right lung weighed 250 grams, congested, some adhesions of pleural surfaces. Heart weighed 310 grams, valves normal. The sinuses of Valsalva were incrusted with calcareous nodules, the aorta dilated at its origin to double the normal size and gradually increased to the beginning of the transverse portion of the areh where the ex pansion was much greater and formed the beginning of a large fusiform aneurism which extended from this point to the lower border of the seventh dorsal vertebra, including the left sides of the bodies of five vertebræ with the heads and necks of the corresponding ribs. The posterior wall of the sac was formed by the exposed bone from the third to the seven th vertebra inclusive. The bodies of all were more or less eroded and the heads and necks of the fourth and fifth ribs had been completely absorbed. The spinal canal was intact. The walls of the sac were thick and firm with deposits of organized clots in places interspersed with plaques of carcareous material. The edges were firmly adherent to the vertebræ and there was no rupture. Capacity of the sac about fifteen hundred cubic centimeters, nearly filled with loose antemortem clots. There was evident pressure on the left vagus nerve.

Case 2.-G. C., aged 57 years, native of the United States, was admitted to the marine ward of the German Hospital, Philadelphia, July 15, 1895 ; died July 19, four days later. Pa tient stated that he had been suffering from asthma (according to his physicians) for nine months constantly, though at times he was better. On examination there was orthopnea, prolonged expiration, wheezing, râles all over chest, respiration weaker in right lung, and occasional cough. Circulation and heart sounds apparently normal, though the examination was not satisfactory owing to the râles and dyspnea. Aneurism was diagnosed as probable, chiefly on account of the persistent dyspnea and the feeble respiration in the right lung. Tracheal tugging was not sought. Of various remedies tried morphia afforded most relief, but the dyspnea continued and the pa tient died from exhaustion.

Necropsy.-.-Heart hypertrophied, especially the left ventricle atheroma of the base of the aortic valves though the valves were competent. The aorta was dilated and from its arch, anteriorly, between the origin of the arteria innominata and left common carotid, arose a saceular aneurism, capacity about four hun dred cubic centimeters, filled with a hard, laminated, grayish clot. The edges of the sac were attached in front to the posterior surface of the manubrium between the firstand second ribs, the bone being eaten through on the right side, but forming scarcely a perceptible bulge in front. The lumen of the aortic arch seemed little, if at all, encroached on, the tumor pressing especially on the arteria innominata, the right bronchus and right pneumogastric nerve. There was no rupture of the sac and death must have been caused by pressure on the vagus nerve.

Case 3 has not yet come to a necropsy. J. B., aged 48 years, native of Maine, a tall, 6 foot 3 inch, powerful- looking man, was admitted to the marine ward of the German Hospital, Philadelphia, Sept. 18, 1895. History : Family history good; admits gonorrhea, denies syphilis: had "rheumatism" in 1866 , but no fever nor swelling of the joints: has had slight cough with expectoration of mucus for three years. For the past eight months says he has suffered from " asthma" which is worse by spells and generally worse on lying down. Three months ago he was taken with pain in the right shoulder, right side of the neck and left knee. Examination: Breathing high pitched over both lungs. Numerous râles, coarse and fine, over both lungs, especially at end of inspiration. A diastolic murmur was heard over the upper part of the sternum, but no thrill nor pulsation could be felt and there was no pain nor swelling. Hard, dry, high-pitched, metallic cough at intervals of half hour or hour, night and day. No inequality of the pupils, but he states that the right side ef his face sweats more than the left. A distinct though slight tracheal tugging is perceptible with each heart pulsation.

The diagnosis was aneurism of the arch of the alorta. October 17, patient asked attention to a swelling behind his left knee which he first noticed three months ago. There was some pain at first but lately he has felt only weakness in the leg.

An aneurism as large as a pigeon's egg was detected in the popliteal space. By an oversight his leg had not been examined before.

October 26, pulsation ean now be felt over the right sternoclavicular articulation and there is unmistakable bulging on this side, thus removing all doubt, if there were any left, as to the correctness of the diagnosis.
The patient was put on the Valsalva-Tufuell treatment but could not stand it and was allowed to get up. The operation of ligation of the subelavian and carotid arteries was explained to him but did not meet his approval and he was discharged at his own request. Now. 4,1895 , not improved.

\section{PURULENT OPHTHALMIA.}

l'repared for the Mitchell Iistrict Medical society of Indiana. Dec. 26,1895

BY DUDLEY S. REYNOLDS, A.M., M.D..

PROFESSOR OF OPHTHALMOLOGY, OTOLOGY AND MEDICAL JURISPRUDENCF IN THE HOSPITAL COLLEGE OF MEDICINE; SURGEON TO THE EYE AND EAR DEPART MENT OT THE LOUISVHLE CITY HOSPITAL, ETC. EOLISVILLE, KY

Purulent conjunctivitis should have a degree of significance to the physician according to the character of the infection and the age of the patient. While there are many varying degrees of intensity, for clinical purposes, it is best to recognize but two causes; the gonorrheal and the endemic.

The gonorrheal is most malignant and intractable; it seldom occurs in both eyes simultaneously. It is most common in adults, and is the result of contagion. The endemic is equally contagious and differs in its clinical features from the gonorrheal type in degree of severity and extent of invasion. In gonorrheal inflammation, the cocci not only attack the mucous corpuscles and surface epithelium, but penetrate quickly into the mucous follicles, which become greatly distended, causing such interruption in the circulation of the blood in the capillary coils surrounding the follicles that great edema is quickly set up, producing a degree of swelling of the ocular conjunctiva often quite sufficient to very nearly overlap the entire cornea, giving to its vertex a decidedly umbilicated appearance.

These phenomena are never seen in the endemic form, which depeuds upon the presence of one or the other of two varieties of staphylococci. The disease in its earliest stages, presents a golden yellow colored pus, or a cream colored stringy muco-pus. In the gonorrheal infection there are always extensive abrasions of the surface in the lining of the lids. In the endemic type this never occurs, and while there are in reality two distinct forms of staphylococci which produce purulent conjunctivitis, one of them stringy, the other golden yellow, there is little difference in degree of severity and in the clinical course of the inflammation. In the new-born, the endemic is the most common type of purulent inflammation, and it is vastly more dangerous to the cornea, than in adults. It is extremely doubtful if any case of gonorrheal conjunctivitis ever resulted from maternal infection in the course of natural delivery. In the first place, as is well known, the skin of the fetus is everywhere covered with the vernix caseosa, the eyes being closed. After the birth of the child, when the eyes are opened, the lash lifts away all external mat. ter, and its arrangement along the free borders of the lids is such as to constitute a practically impassable barrier for any matter that might be lodged upon the skin, even if it be of a fluid nature. When the nurse undertakes the removal of the cheesy coating from the skin, the eyes are frequently inoculated with whaever matter may be on the rag or sponge. Staphylococci are nearly constantly present about the finger nails and sponges handled by untidy persons; and simple muco purulent vaginitis, with which the mother or nurse may be suffering, constitutes a vastly more common source of inoculating 